\title{
AVALIAÇÃO DA ADESÃO AO TRATAMENTO DO DIABETES MELLITUS TIPO 1: REVISÃO DE LITERATURA
}

\author{
Luziane Fatima Kirchner \\ Mestre em Análise do Comportamento pela Universidade Estadual de Londrina. Doutoranda em \\ Psicologia pela Universidade Federal de São Carlos - SP. \\ Maria Luiza Marinho-Casanova \\ Doutora em Psicologia Clínica pela Universidade de São Paulo e professora do Departamento de \\ Psicologia Geral e Análise do Comportamento da Universidade Estadual de Londrina - PR.
}

\begin{abstract}
Resumo
Esse trabalho buscou identificar os métodos, objetivos e tipo de estudos que avaliaram a adesão ao tratamento do Diabetes Mellitus tipo 1, publicados entre 2000 e 2013. Foram analisados 111 artigos, levantados na base de dados Web of Science. Os resultados indicaram que 95 pesquisas são descritivas, com participantes predominantemente adolescentes (74 estudos) e crianças (34 estudos). Verificou-se também que prevaleceram aqueles estudos que: utilizaram apenas um informante (67 estudos), sendo o portador da doença quem mais informou os dados (72 estudos); foram avaliados de um (35 estudos) ou cinco (25 estudos) componentes do tratamento médico, por meio de um único instrumento de avaliação (68 estudos), sendo os instrumentos padronizados a medida de avaliação mais utilizada (40 estudos). Os dados levam ao questionamento de que os métodos utilizados para avaliar a adesão, nos estudos levantados, não estão de acordo com sugestões apresentadas na literatura para tornar resultados da avaliação da adesão mais confiáveis. Discussões a respeito desta temática precisam ser mais desenvolvidas.

Palavras-chave: adesão ao tratamento; revisão de literatura; diabetes mellitus tipo 1.
\end{abstract}

\section{EVALUATION OF ADHERENCE TO THE TREATMENT OF TYPE 1 DIABETES MELLITUS: LITERATURE REVIEW}

\begin{abstract}
This work aimed to identify methods, objectives and type of studies that assessed adherence to the treatment of type 1 Diabetes Mellitus, published from 2000 to 2013. We analyzed 111 articles, collected in the survey by the Web of Science database. The results indicated that 95 studies are descriptive, with prevalence of adolescents (74 studies) and children (34 studies). It was also found that those studies that have prevailed: Used an informer (67 studies), the carrier of the disease who reported more data (72 studies), evaluated one (35 studies) or five (25 studies) treatment medical, by means of an instrument (68 studies), being standardized instruments the most used procedures in this assessment (40 studies). Data leaded to the conclusion that the methods used to assess adherence, in the studies analyzed, do not comply with the suggestions made in Literature to make the results in adherence more reliable. Discussions about this subject need to be further developed.
\end{abstract}

Keywords: adherence to treatment; literature review; type 1 diabetes mellitus. 


\title{
EVALUACIÓN DE LA ADHERENCIA AL TRATAMIENTO DE LA DIABETES MELLITUS TIPO 1: REVISIÓN DE LA LITERATURA
}

\begin{abstract}
Resumen
Este estudio trata de identificar los métodos, los objetivos y el tipo de estudios que evaluaron la adherencia al tratamiento de la Diabetes Mellitus tipo 1, publicados entre 2000-2013. Se analizaron los artículos 111, planteada por la bases de datos Web of Science. Los resultados indicaron que 95 estudios son descriptivos, con participantes predominantemente adolescentes (74 estudios) y los niños (34 estudios). También había prevalecido esos estudios que utilizan un informante (67 estudios), siendo el portador de la enfermedad que los datos más informo (72 estudios); evaluado una (35 estudios) o cinco ( 25 estudios) los componentes de los tratamientos médicos, a través de un instrumento de evaluación (68 estudios), siendo los instrumentos estandarizados más comúnmente utilizados (40 estudios). Los datos conducen el cuestionamiento que los métodos utilizados para evaluar la adherencia, en los estudios examinados, no están de acuerdo con las sugerencias hechas en la literatura para hacer que los resultados de evaluación de la adherencia sean más fiables. Las discusiones sobre este tema es necesario desarrollar aún más.

Palabras clave: adherencia al tratamiento; revisión de la literatura; diabetes mellitus tipo 1.
\end{abstract}

\section{INTRODUÇÃO}

A não adesão ao tratamento de doenças crônicas pode afetar de forma adversa a saúde e o bem estar do indivíduo, bem como a relação custo-benefício de cuidados médicos, elevando os gastos para a população e para o Estado, com desperdício de medicamentos, aumento de internações e atendimentos ambulatoriais (Rapoff, 2009). Aderir ao tratamento médico pode ser entendido por Haynes et al (2008) como o grau de conformidade entre as recomendações dos profissionais de saúde e o comportamento da pessoa direcionado a executar o tratamento proposto. O tipo do tratamento vai depender da gravidade da doença, das características do portador e do seu estado de saúde atual (Arruda \& Zannon, 2002). Para o Diabetes Mellitus tipo 1, cuja doença refere-se a destruição de células beta (que produzem a insulina responsável pelo controle de açúcar no sangue) pelo próprio organismo, o tratamento envolve a aplicação da insulina (fabricada em laboratório) e o controle do nível de glicose no sangue. $O$ Tipo, a dose e frequência da aplicação de insulina variam de um indivíduo para outro, dependendo das características da doença e da manutenção dos cuidados com a saúde, como alimentação e atividade física (Costa \& Neto, 2004).

No caso de uma doença crônica, como o diabetes, avaliar a adesão ao tratamento envolve mensurar as dimensões com que o portador da doença executa cada componente do tratamento prescrito e, a partir disso, utilizar critérios para classificar os pacientes que aderem ou não (Quittner, Modi, 
Lemanek, Ievers-Landis, \& Rapoff, 2008). Para este estudo, "dimensão" referese às unidades de medida consideradas na avaliação de um componente do tratamento (por exemplo, intensidade, frequência e/ou duração na execução de um componente), e componente do tratamento" corresponde à sequência de cuidados a serem administrados (por exemplo, dieta, aplicação da insulina, controle glicêmico, atividade física, medicação, entre outros).

Existem diferentes instrumentos de medidas para avaliá-la, que podem ser classificados em diretos ou indiretos (Balkrishnan \& Jayawant, 2007). Os instrumentos de medida direta geralmente são de alto custo, mas contam com a precisão dos dados objetivos. Esses podem ser, por exemplo, o monitoramento eletrônico e os indicadores biológicos (ex: exames laboratoriais). Os instrumentos de medida indireta se referem à obtenção da informação do nível de adesão por meio do relato dos portadores da doença, como os autorrelatos e os autorregistros. Esses últimos são mais baratos, porém, estão sujeitos ao viés da informação fornecida pelos participantes, que são capazes de superestimar os resultados na tentativa de evitar a desaprovação médica pela não adesão (Bosworth, 2005: Balkrishnan \& Jayawant, 2007). De acordo com Smith, Hankins, Hodson e George (2010) uma maneira de evitar este viés, é que mais pessoas, além do portador da doença, prestem os mesmos tipos de informação (pais, médicos, enfermeiras, professores, entre outros), a fim de poder cruzar os dados obtidos ou conseguir informações sob outro ângulo de observação.

Dependendo da finalidade da avaliação, um instrumento pode ser preferível em relação a outro. O que deve ser ponderado na escolha do instrumento é a conveniência e a aceitabilidade do participante quanto a maneira de avaliação, o custo financeiro e a abrangência do instrumento para o que se pretende estudar (Vitolins, Rand, Rapp, Ribisl, \& Sevicky, 2000; Quittner, Espelage, Ievers-Landis, \& Drotar 2000). Segundo Quittner et al, (2008), a escolha do instrumento não é simples, uma vez todos os instrumentos apresentados na literatura são questionáveis quanto à possibilidade de avaliar a adesão do indivíduo a todos os componentes do tratamento médico e todas as suas dimensões. Ao observar as limitações quanto à abrangência de um instrumento, outros cuidados devem ser tomados na avaliação, como escolher apenas alguns componentes para avaliar ou envolver menor quantidade de participantes (Gonzalez \& Schneider, 2011).

Rudd (1979) relata que os pesquisadores na década de 70 buscavam um "padrão-ouro para medir a adesão". Após quase 32 anos, apesar do avanço 
tecnológico e muitos estudos empíricos desenvolvidos, Gonzalez \& Schneider (2011) apontam que a questão sobre qual o melhor método para avaliar a adesão continua em aberto. No Brasil, os estudos sobre adesão ao tratamento do Diabetes Mellitus tipo 1 são descritivos (Bloch, Melo, \& Nogueira, 2008; Souza, Silva, Freitas, \& Borges, 2014), ou apresentam de maneira não sistematizada os métodos avaliação da adesão (Leite \& Vasconcellos, 2003; Dias et al, 2011). Entretanto, revisões sistemáticas sobre este tema não foram encontradas. Tal aspecto impulsionou a condução da presente pesquisa: realizar uma revisão de estudos publicados em um período de 13 anos (de 2000 a 2013), que avaliaram a adesão ao tratamento do Diabetes Mellitus tipo 1, identificando o método, objetivo e tipo de estudo de cada publicação.

\section{MÉTODO}

Os documentos selecionados para o estudo foram artigos disponibilizados na íntegra pelo Portal de Periódicos Capes, na base de dados Web of Science, que tiveram como objetivo principal avaliar a adesão de portadores do Diabetes Mellitus tipo 1, a um ou mais componentes do tratamento médico recomendado (ex.: seguir dieta alimentar, fazer atividades físicas, controlar a glicemia, entre outros). A busca foi realizada no período de janeiro de 2014 , de estudos que foram publicados no período de 2000 a 2013.

Para a estratégia de busca foram utilizadas as palavras, "diabetes AND adherence OR nonadherence OR compliance OR noncompliance NOT type 2 OR gestational", que deveriam constar no título do artigo. Não houve restrição quanto à área estudada, o que permitiu identificar as medidas de avaliação da adesão adotadas por pesquisadores de diversas áreas.

As referências bibliográficas dos estudos levantados foram armazenadas no programa EndNote $X 4 \AA$ (versão para desktop) e os dados dos estudos foram arquivados em sete campos do programa Microsoft Office Access 2007, criados especificamente para este estudo: tipo de pesquisa (descritiva - não envolve a manipulação de variáveis, quase-experimental - envolve a manipulação de variáveis), objetivos dos estudos (foram agrupados em categorias que descrevem a variável estudada em relação a adesão: relação familiar, indicadores de saúde, atuação médica, características sociodemográficas/psicossociais do portador da doença, ou efeito do tratamento), dados sobre a população portadora do diabetes (idade e quantidade 
de indivíduos portadores do diabetes), fontes de informação sobre a adesão (profissional de saúde, mãe/pai/cuidador, registros de prontuários ou de farmácia, e/ou o próprio portador da doença), componente de tratamento avaliado (atividade física, consulta médica, insulina, dieta, medicação, glicemia, e/ou outro), e instrumentos adotados na avaliação da adesão (protocolo de observação do comportamento, registro de farmácia, roteiro de entrevista/questionário, registro de prontuário médico e/ou instrumento padronizado), em cada estudo.

\section{RESULTADOS}

Foram levantados e analisados 111 estudos que avaliaram a adesão ao tratamento do Diabetes Mellitus tipo 1, dentre os quais 95 foram estudos descritivos, e 16 estudos quase experimentais. No que se refere aos objetivos, 21 estudos investigaram aspectos da relação familiar (ex: mãe, pai, cônjuge, outros cuidadores) com a adesão, e 20 correlacionaram variáveis sociodemográficas (ex: idade, sexo, gênero) ou psicossociais (ex; autoestima, autoeficácia, depressão, estresse), com a adesão ao tratamento médico.

Houve predomínio de estudos envolvendo grande quantidade de diabéticos (111 a 1.000 indivíduos, 56 estudos), sendo também expressiva a frequência de estudos com 11 e 100 indivíduos (26 estudos). Os estudos descritivos foram os que envolveram a maior quantidade de diabéticos, sendo que um deles (Yang et al., 2009) apresentou os dados de 1.888 .682 pessoas. A faixa etária foi identificada em 91 estudos e os outros estudos $(n=20)$ não apresentaram esta informação. Houve predomínio de estudos com adolescentes diabéticos, entre 12 a 18 anos (74 estudos), e estudos com crianças de 0 a 11 anos (44 estudos). Estudos realizados com população adulta ou idosos (acima de 65 anos) foram menos frequentes (34 e 28 estudos, respectivamente).

A análise dos estudos levantados indicou que nem sempre foram os portadores do diabetes que informaram os dados sobre a sua adesão ao tratamento. A Tabela 1 apresenta as fontes de informação sobre a adesão, podendo ser o próprio indivíduo e/ou os dados foram retirados de outras fontes. 
Tabela 1.

Informantes dos dados sobre a adesão ao tratamento (111 estudos).

\begin{tabular}{|c|c|c|c|c|c|}
\hline $\begin{array}{c}\mathrm{N}^{\mathrm{O}} \mathrm{de} \\
\text { informantes }\end{array}$ & $\begin{array}{l}\text { Profissional } \\
\text { de saúde }\end{array}$ & $\begin{array}{l}\text { Mãe/Pai/ } \\
\text { cuidador }\end{array}$ & $\begin{array}{c}\text { Reg. } \\
\text { Prontuário } \\
\text { /farmácia }\end{array}$ & $\begin{array}{l}\text { Portador } \\
\text { da } \\
\text { doença }\end{array}$ & $\begin{array}{c}\text { Freq. de } \\
\text { estudos por } \\
\text { quantidade de } \\
\text { informante (s) }\end{array}$ \\
\hline 1 informante & 1 & 7 & 26 & 33 & 67 \\
\hline 2 informantes & 5 & 20 & 18 & 33 & 38 \\
\hline 3 informantes & 3 & 4 & 5 & 6 & 6 \\
\hline $\begin{array}{l}\text { Freq. de estudos } \\
\text { por tipo de } \\
\text { informante }\end{array}$ & 9 & 31 & 49 & 72 & 111 \\
\hline
\end{tabular}

Predominaram os estudos nos quais os dados foram coletados por apenas um informante (67 estudos), sendo as principais fontes de informação, o portador da doença (33 estudos) ou os registros obtidos de prontuários médicos/farmácia (26 estudos). Tais registros correspondem as informações de uma base de dados eletrônica que calcula a diferença entre a data e a quantidade de medicamentos retirados na farmácia pelo portador da doença, e a data e quantidade de medicamentos que deveriam ser retirados de acordo com a prescrição médica). Quando foram dois informantes (38 estudos), a tabela indica a predominância de portadores do diabetes (33 estudos), cuidadores (20 estudos) e registros de prontuários/farmácia (18 estudos). Poucos estudos coletaram dados com três informantes (6 estudos), e para esses estudos os resultados foram similares no que se refere ao tipo de informante.

A Tabela 2 apresenta informações sobre os componentes do tratamento médico avaliados como forma de indicar dados sobre a adesão. Uma pequena parcela dos estudos $(n=2)$ não identificou quais componentes foram avaliados, e foi excluída desta análise. 
Tabela 2.

Componente(s) do tratamento médico avaliado(s) nos estudos (109 estudos).

\begin{tabular}{|c|c|c|c|c|c|c|c|c|}
\hline $\begin{array}{c}\text { No } \\
\text { componentes } \\
\text { avaliados }\end{array}$ & $\begin{array}{l}\text { Atividade } \\
\text { física }\end{array}$ & $\begin{array}{l}\text { Consulta } \\
\text { médica }\end{array}$ & Insulina & Dieta & Medicação & Glicemia & Outro & $\begin{array}{c}\text { Freq. de } \\
\text { estudos por } \\
\text { quantidade de } \\
\text { componente(s) }\end{array}$ \\
\hline 1 & 1 & 1 & 1 & 3 & 14 & 10 & 2 & 35 \\
\hline 2 & 3 & 3 & 3 & 6 & 3 & 12 & 5 & 19 \\
\hline 3 & 2 & 0 & 2 & 3 & 2 & 2 & 2 & 4 \\
\hline 4 & 13 & 1 & 16 & 16 & 2 & 16 & 5 & 19 \\
\hline 5 & 21 & 1 & 22 & 22 & 3 & 23 & 23 & 25 \\
\hline 6 & 6 & 2 & 5 & 6 & 5 & 6 & 6 & 7 \\
\hline $\begin{array}{l}\text { Freq. de } \\
\text { estudos por } \\
\text { componente } \\
\text { avaliado }\end{array}$ & 50 & 8 & 54 & 62 & 30 & 76 & 48 & 109 \\
\hline
\end{tabular}

Como mostra a Tabela 2, predominaram os estudos que avaliaram 1 ou 5 componentes do tratamento para o diabetes, respectivamente, 35 e 25 estudos. Para os estudos que buscaram medir a adesão a um único componente do tratamento, a medicação (14 estudos) e a glicemia (10 estudos) foram os componentes mais avaliados. E para os estudos que avaliaram 5 componentes do tratamento, os estudos mediram predominantemente a glicemia, dieta, insulina, atividade física e outros (ex.: reações do indivíduo em situações de hipoglicemia ou hiperglicemia, exame dos pés e dos olhos, avaliação do peso corporal, exame da pressão arterial).

As dimensões atribuídas para classificar os níveis de adesão, em cada componente, foram muito variadas entre os estudos, de forma que foi difícil quantificá-las na presente pesquisa. Entretanto, foi possível identificar que 58 de 109 estudos avaliaram o(s) componente(s) do tratamento considerando apenas uma dimensão para cada componente. Para os componentes mais avaliados (glicemia, dieta e insulina), as principais dimensões consideradas foram: a) glicemia: frequência e nível da taxa glicêmica medida em casa ou resultados obtidos pelo exame Hba1c, b) dieta: quantidade de refeições realizadas ou quantidade de carboidratos consumidos ao dia, c) insulina: horário e a frequência de aplicação ou dose de insulina aplicada. 
$\mathrm{Na}$ revisão realizada, foi possível identificar quais foram os instrumentos utilizados para coletar os dados sobre a adesão. A Tabela 3 apresenta a frequência de estudos que utilizaram um, dois ou três instrumentos.

Tabela 3.

Instrumento de avaliação da adesão (100 estudos).

\begin{tabular}{|c|c|c|c|c|c|c|c|}
\hline $\begin{array}{c}\mathrm{N}^{\circ} \mathrm{de} \\
\text { instrumentos }\end{array}$ & $\begin{array}{l}\text { Protocolo de } \\
\text { observação do } \\
\text { comportamento }\end{array}$ & $\begin{array}{l}\text { Registro } \\
\text { de } \\
\text { farmácia }\end{array}$ & $\begin{array}{l}\text { Roteiro de } \\
\text { entrevista/ } \\
\text { questionário }\end{array}$ & $\begin{array}{l}\text { Protocolo de } \\
\text { autorregistro }\end{array}$ & $\begin{array}{l}\text { Registro } \\
\text { de } \\
\text { prontuário } \\
\text { médico }\end{array}$ & $\begin{array}{l}\text { Instrumento } \\
\text { padronizado }\end{array}$ & $\begin{array}{c}\text { Freq. de } \\
\text { estudos por } \\
\text { quantidade } \\
\text { de } \\
\text { instrumentos }\end{array}$ \\
\hline 1 & 1 & 4 & 14 & 9 & 17 & 23 & 68 \\
\hline 2 & 1 & 5 & 5 & 13 & 16 & 14 & 27 \\
\hline 3 & 0 & 3 & 1 & 3 & 5 & 3 & 5 \\
\hline $\begin{array}{c}\text { Freq. de } \\
\text { estudo por } \\
\text { instrumento }\end{array}$ & 2 & 12 & 20 & 25 & 38 & 40 & 100 \\
\hline
\end{tabular}

Foi predominante o número de estudos que utilizou apenas um instrumento para medir a adesão (68 estudos). Para as pesquisas que utilizaram um ou dois instrumentos, os instrumentos padronizados e registro de prontuário médico foram os mais escolhidos. Apenas 5 estudos utilizaram três instrumentos para coletar os dados sobre a adesão. Um exemplo é o estudo de Williams et al, (2009), que utilizou registro de farmácia, roteiro de entrevista com os portadores da doença e registro de prontuário médico (exame laboratorial Hba1c) para avaliar a adesão à medicação de 2.973 diabéticos.

\section{DISCUSSÃO}

O presente estudo fornece evidências de que embora a adesão ao tratamento ainda seja vista como "o seguimento de prescrições médicas", novas discussões sobre esse conceito são construídas a partir de cada estudo, e se iniciam com a escolha dos componentes do tratamento médicos a serem avaliados e os métodos empregados na avaliação (Clifford, Perez-Nieves, Skalicky, Reaney, \& Coyne, 2013). A presente pesquisa teve por objetivo apresentar a diversidade dos métodos utilizados em estudos empíricos que avaliam a adesão, e para isso abrangeu 111 estudos realizados em diferentes áreas da saúde, com portadores do Diabetes Mellitus tipo 1, de todas as faixas etárias. 
Os estudos tiveram como população predominante as crianças e adolescentes, e embora tenham relacionado diversas variáveis à adesão, a mais estudada foi o impacto da relação familiar na adesão, sobretudo para as crianças e os adolescentes diabéticos e seus familiares. Os principais dados levantados são condizentes com afirmativas apontadas na literatura, as quais indicam que o Diabetes Mellitus tipo 1 é uma doença crônica que tem maior incidência na população infantil e de adolescentes (Patton,2006) e que estudos envolvendo a interação familiar são prevalentes, devido a essa população envolver a participação dos pais como mediadores na adesão (Mackey \& La Greca, 2007).

A adesão ao tratamento de doenças crônicas não está delimitada a padrões fixos de comportamentos a serem seguidos, pois diante do mesmo diagnóstico, os sintomas apresentados pelo paciente são os que estabelecem os critérios para as recomendações médicas (Jonhson, 1992; Arruda \& Zannon, 2002). Essa afirmativa mostra a importância de se considerar as variáveis individuais na avaliação da adesão, compreendendo que o tratamento tem implicações diferentes para cada indivíduo. No entanto, algumas pesquisas levantadas parecem não ter tomado esses cuidados, ao descrever os dados da população estudada. Verificou-se que 20 estudos não identificaram a faixa etária da população diabética, e muitos envolveram, de forma geral, grande quantidade de participantes (até no máximo 1.888 .682 pessoas). A amostra foi de 111 a 1.000 indivíduos para 56 estudos e 11 a 100 para 26 estudos, sendo prevalentes as pesquisas descritivas (95 estudos). Em relação a isso, Sackket (1976) pontua que pesquisas que envolvem grande quantidade de participantes, geralmente realizadas em centros de referência médica, tomam como base de avaliação poucas dimensões do tratamento (exemplo: mede a frequência diária de aplicação da insulina, mas não o horário e se a dose aplicada esta adequada a taxa glicêmica) e por esse motivo podem tendenciar os dados relativos à adesão.

Para Cozby (2003), deve-se considerar que a probabilidade de uma amostra fornecer dados que reflitam o valor real da população, aumenta com o aumento do tamanho da amostra. No entanto, essa afirmativa é valida quando se pretende avaliar medidas objetivas e relativamente estáveis. No caso da adesão, cujas recomendações médicas e as respostas ao tratamento são diferenciadas para cada indivíduo, a avaliação deve envolver menor quantidade de participantes e abranger as medidas do tratamento de forma peculiar (Quittner et al, 2000). 
Outra questão que pode interferir na confiabilidade dos dados refere-se à quantidade de fontes utilizadas para fornecer os dados a respeito da adesão do portador da doença. Para isso, Smith et al, (2010) sugerem que mais de uma fonte seja consultada. Nos estudos levantados, houve predomínio daqueles que coletaram dados com apenas um informante (68 estudos). Para os estudos que utilizaram dois ou três informantes (38 e 6 estudos, respectivamente), a maior parte deles mostrou adequar-se às sugestões apresentadas por Quittner et al, (2008), de utilizar mais de um informante para obter dados mais precisos sobre o mesmo componente do tratamento médico. Holmbeck et al, (1998) alertam que usar vários informantes, para avaliar a adesão, não é uma tarefa fácil. Esses autores avaliaram a adesão da criança ao tratamento da espina bífida por meio de múltiplos informantes (pai, mãe, professor e profissional de saúde) e verificaram que eles apresentaram dados contraditórios, ou seja, enquanto os pais relatavam baixa adesão, os professores relatavam que a criança aderia a todos os componentes do tratamento. Jonhson (1995) justifica essa discrepância entre os dados, afirmando que cada respondente tem acesso ao comportamento da criança em um contexto diferente, e, portanto, relatam somente aquilo que observam.

Os componentes do tratamento mais avaliados pelos estudos levantados, de forma geral, foram: glicemia, dieta e insulina, e acredita-se que predominaram por serem os componentes do tratamento mais difundidos nos estudos sobre Diabetes Mellitus tipo 1 (Organização Mundial de Saúde, 2003). O componente mais avaliado isoladamente foi a medicação (14 estudos), sendo medida nesses estudos por uma única dimensão: o cálculo da quantidade de medicação obtida na farmácia, dividido pelo número de dias prescritos para o uso, ou o calculo de "interrupções" ou "dias de atraso" do indivíduo para buscar o remédio na farmácia. Karve et al, (2008) pontua que essa forma de medir a adesão à medicação ganhou destaque nos últimos anos, pois permite avaliar um grande número de participantes, sem que eles tenham o conhecimento explícito de estarem sendo avaliados, minimizando os problemas de reatividade. Em contrapartida, esta medida parece estar muito distante de avaliar o comportamento de adesão, pois comprar a medicação prescrita não envolve necessariamente tomá-la na quantidade, dia e horário recomendado (Cleemput, Katrien Kesteloot, \& DeGeest, 2002). 
Cada um dos componentes do tratamento médico recomendado é complexo e abrange dimensões que devem estar claramente definidas no momento da avaliação (Quittner et al, 2000). Por exemplo, a avaliação da adesão à atividade física não pode considerar apenas a frequência com que o indivíduo se exercita, mas também as outras dimensões do exercício, como a intensidade e a duração. Devido à complexidade na avaliação de cada medida, Jhonson (1993) sugere ser mais apropriado avaliar um único componente do tratamento e abranger todas as suas dimensões.

Um estudo realizado por Keulen, Mesters, Mechelen e Vries (2010) comparou níveis de adesão por meio da avaliação de um único componente do tratamento e de vários componentes do tratamento médico. Os autores verificaram que quando são avaliados vários componentes do tratamento, os participantes tendem a emitir respostas globais sobre os seus comportamentos e a superestimar os níveis de adesão. Os autores discutem que os instrumentos que avaliam múltiplos componentes não conseguem abranger as dimensões de todos eles, oferecendo dados imprecisos sobre a adesão.

Dos 111 estudos levantados, verificou-se que 35 estudos avaliaram um único componente do tratamento e um número expressivo de estudos $(n=25)$ avaliou cinco desses componentes, demonstrando que mais discussões sobre a avaliação da adesão ao tratamento médico precisam ser desenvolvidas, a fim de esclarecer a necessidade de avaliar um componente por vez. O presente trabalho realizou uma análise da frequência dos componentes do tratamento abordados, mas não se ateve a observar cautelosamente a forma como cada componente foi avaliado, ou seja, suas dimensões. Pesquisas com este objetivo também precisam ser desenvolvidas.

De acordo com Rand (1990), é necessário ter clareza de quais componentes do tratamento e dimensões serão consideradas, para posteriormente selecionar instrumentos mais apropriados para avaliá-los. Rapoff (2009), Vitolins et al, (2000) e Quittner et al, (2000) pontuam que não há critérios para optar em avaliar um ou outro componente do tratamento médico, e a seleção do instrumento de avaliação dependerá da possibilidade de abrangência do mesmo para o(s) componente(s) e as dimensões que se pretende avaliar, o seu custo financeiro e a aceitabilidade do participante.

Os instrumentos padronizados foram os instrumentos de avaliação mais utilizados, isoladamente ou em conjunto com outros (40 estudos). Autores 
sugerem que, de fato, esses instrumentos sejam os mais adotados entre as pesquisas que avaliam a adesão, por diversas vantagens: ser simples e barato para avaliar a adesão, ser facilmente incorporado à prática clínica (Bosworth, 2005; Rapoff, 2009), apresentar categorias de avaliação padronizadas e préestabelecidas (Shiling, Grey, \& Knalf, 2002), e ter alcançado progressos quanto à validação (Rapoff, 2009). As desvantagens dessa medida é que ela está sujeita ao viés da informação, pode gerar problemas para recordar situações passadas (ex: quantas vezes você tomou a medicação esta semana?), envolver geralmente perguntas globais sobre a adesão (ex: qual a porcentagem de dias, ao longo dos últimos 6 meses, que você tomou o remédio prescrito?), e ser limitada quanto à avaliação de múltiplas dimensões dos componentes do tratamento avaliados (Shiling, Grey, \& Knalf, 2002).

A outra fonte de informação mais frequente nos estudos levantados foi 0 registro de prontuário médicos (38 estudos). As informações obtidas com esse registro apoiaram-se predominantemente em dados de exames laboratoriais (ex.: HBa1c, perfil lipídico) ou clínicos (ex.: avaliação médica de complicações renais ou oculares). Para Jonhson (1992), os resultados de saúde são comumente utilizados como medidas de adesão, sugerindo que o bom controle metabólico indica melhores níveis de adesão.

De acordo com Nemes, Helena, Caraciolo, \& Basso (2009) resultados de saúde e adesão não são a mesma coisa, mas os dados das duas medidas são utilizados para estabelecer importantes correlações, validar medidas de adesão ou até mesmo constituir pontos de corte entre indivíduos que aderem ou não. Em alguns estudos levantados, o nível glicêmico foi atribuído explicitamente, pelos autores, como forma de medir a adesão (Haler, Gil, Gardner, \& Whittier, 2009; Manzano et al, 2006) e para outros estudos, este dado foi incluído em pesquisas nas quais não foram feitas distinções entre medidas de adesão e resultados de saúde (Kirkman, Williams, Caffrey, \& Marrero, 2002; Lewin et al, 2005). DiMatteo et al, (2002) e Moraes, Rolim e Costa-Junior (2009) sugerem que embora sejam formas práticas para se obter resultados objetivos, os resultados de saúde e a adesão devem ser avaliados de forma independente, pois podem sofrer alterações devido a variações genéticas ou reações adversas aos medicamentos, além de não envolverem o estudo do comportamento de adesão propriamente dito. 
A observação direta, mesmo sendo a medida mais indicada para avaliar a adesão, por possibilitar avaliações de diferentes dimensões do comportamento (como frequência, duração, desempenho, entre outras) e revelar algumas variáveis (antecedentes e consequentes) do comportamento estudado (Rapoff, 2009), foi a menos utilizada nos estudos $(n=2)$. Os estudos que utilizaram esta medida realizaram as observações durante as refeições, na casa dos participantes (Patton, Dolan, \& Powers, 2006; Pantton, Piazza-Waggoner, Modi, Dolan, \& Powers, 2009). Freeman (2008) pontua que, por depender da disponibilidade dos participantes e da permissão para serem observados, esta é a forma de avaliação mais dificilmente executada.

Não se pretende discutir detalhadamente todos os instrumentos de avaliação adotados nos estudos levantados, mas a necessidade de utilizar mais de um instrumento ao avaliar a adesão. Como comentado anteriormente, Rand (1990), Vitolins et al, (2000), Patton (2006) e Rapoff (2009) sugerem utilizar dois ou mais instrumentos para avaliar a adesão, permitindo que as forças de um instrumento possam compensar as fraquezas do outro, e capturar de forma mais precisa as informações necessárias para determinar os níveis de adesão.

Para Rapoff (2009) a comparação de dados entre instrumentos pode ser uma forma de minimizar os problemas de validade e confiabilidade na avaliação da adesão. Asche, LaFleur e Conner (2011) sugerem que os pesquisadores comparem instrumentos de medidas diretas (ex.: registro da frequência e quantidade de insulina) e instrumentos de medidas indiretas (ex: autorrelato sobre a aplicação da insulina), mas alertam que mesmo tomando tais precauções, não estarão livres de erros quanto à avaliação da adesão.

DiMatteo (2004) discute a dificuldade em comparar os dados entre dois ou mais instrumentos de avaliação, pois mesmo avaliando um único componente do tratamento, as unidades de medida são diferentes para cada instrumento e apontam diferentes taxas de respostas da adesão. Para ele, a comparação empírica de instrumentos de avaliação da adesão continua sendo um desafio para a área. Os estudos de Smith et al, (2010) e Muller et al, (2011), comprovaram empiricamente essa afirmativa. Ambos os estudos compararam instrumentos de medida indireta (entrevistas e contagens de comprimidos) com instrumentos de medida direta e verificaram grande discrepância entre os dados, com os instrumentos de medida direta indicando menores níveis de adesão. 
Segundo Patton (2006) concepções a respeito da adesão mudaram ao longo dos anos. O que era visto como regra do tratamento a ser seguido, atualmente é observado como uma ampla classe de comportamentos relacionados a diversos cuidados com a saúde (tomar insulina, seguir a dieta recomendada, etc.), determinados por múltiplos fatores (o estado de saúde do indivíduo, os custosbenefícios para seguir um ou outro componente do tratamento médico, entre outros).

Do ponto de vista metodológico, o conceito de adesão é variável e dependerá da forma como ela é avaliada, isto é, quem são os informantes dos dados, quais as variáveis e as dimensões medidas e quais os instrumentos de avaliação utilizados. A complexidade em acessar dados relativos a adesão alerta sobre a preferência de se avaliar os comportamentos de adesão de forma situacional e continuada, sem comparações entre os dados, tomando por base o indivíduo como controle de si mesmo (Quittner et al, 2008).

\section{CONSIDERAÇÕES FINAIS}

O presente estudo buscou identificar os métodos, objetivos e tipo de estudos que avaliaram a adesão ao tratamento do Diabetes Mellitus tipo 1 , publicados entre 2000 e 2013. Foi realizada uma análise dos estudos levantados na Web of Science à luz das estratégias apresentadas na literatura como mais adequadas para avaliar a adesão ao tratamento médico. Sackett (1976), Rand (1990) e Rapoff (2009) consideram que os pesquisadores devem avaliar, em cada estudo, um único componente do tratamento médico, possibilitando assim abranger a avaliação de todas as dimensões deste componente; deve envolver mais de um informante, e adotar mais de um instrumento nesta avaliação.

Os resultados mostraram que todos os estudos levantados estão em desacordo, em pelo menos uma das sugestões apresentadas. No entanto, a análise da literatura justifica que não é fácil avaliar a adesão de acordo com essas estratégias. Embora teoricamente elas sejam aceitáveis, dados de estudos empíricos (Smith et al, 2010; Muller et al, 2011) comprovam que os resultados dos níveis de adesão são discrepantes para múltiplos informantes ou múltiplos instrumentos de avaliação. Isso pode fazer com que os pesquisadores distanciem cada vez mais os métodos empregados das estratégias sugeridas na literatura.

A dificuldade não representa um impedimento para estudos mais completos, mas alerta futuros pesquisadores para o custo de resposta e para a 
necessidade de prever mais recursos quando pretendem avaliar a adesão, de forma que suas pesquisas atendam as exigências de qualidade, podendo fornecer informações úteis e confiáveis para o manejo do diabetes.

\section{REFERÊNCIAS}

Arruda, P.M., \& Zannon, C.M.L.C. (2002) Adesão ao tratamento pediátrico da doença crônica: Evidenciando o desafio enfrentado pelo cuidador. Santo André: Esetec Editores Associados.

Asche, C., LaFleur, J., \& Conner, C. (2011). A review of diabetes treatment adherence and the association with clinical and economic outcomes. Clinical Therapeutics, 33(1), 74-109.

Balkrishnan, R. \& Jayawant, S.S (2007). Medication adherence research in populations: Measurement issues and other challenges. Clinical Therapeutics, 29(6), 61-64.

Bloch, K.V., Melo, A.N., Nogueira, A.R. (2008). Prevalência da adesão ao tratamento anti-hipertensivo em hipertensos resistentes e validação de três métodos indiretos de avaliação da adesão. Cadernos de Saúde Pública, 24(12), 2979-84. Recuperado em 13 de novembro de 2013. Obtido em http://www.scielo.br/pdf/csp/v24n12/30.pdf

Bosworth, B.H. (2005). Patient treatment adherence concepts, interventions and measurement. New Jersey: Lawrence Erlbaum Association.

Cleemput, I., Kesteloot, K., \& DeGeest, S. (2002). A review of the literature on the economics of noncompliance: Room for methodological improvement. Health Policy, 59, 65-94.

Clifford, S., Perez-Nieves, M., Skalicky, A., Reaney, M.D., \& Coyne, K.S. (2013). A systematic literature review of methodologies used to assess medication adherence in patients with diabetes. Value in Health, 16(3), 167-168.

Costa, A.A., \& Neto, J.S.A. (2004). Manual do diabetes: Educação, alimentação, medicamentos e atividade física. ( $4^{a}$ ed.). São Paulo: Savier.

Cozby, P.C. (2003). Métodos de pesquisa em ciências do comportamento. Tradução de: Gomide, Paula Inez Cunha. Otta, Emma. São Paulo: Atlas.

Dias, A. M., Cunha, M., Santos, A., Neves, A., Pinto, A., Silva, A, Castro, S. (2011) Adesão ao componente terapêutico na doença crônica: Revisão da literatura. Millenium, 40, 201 - 219. Recuperado em 13 de novembro de 2011. Obtido em http://www.ipv.pt/millenium/Millenium40/14.pdf 
DiMatteo, M.R. (2004). Variations in patients' adherence to medical recommendations: A quantitative review of 50 years of research. Medical Care, 42(3), 200-209.

DiMatteo, M.R., Giordani, P.J., Lepper, H.S., \& Croghan, T.W. (2002) Patient adherence and medical treatment outcomes: A meta-analysis. Medical Care, $40(9), 794-811$.

Freeman, D. (2008). Cognitive therapy for paranoid and suspicious thoughts. Geriatric Medicine, 38, 28-32.

Gonzalez, J.S., \& Schneider, H.E (2011). Methodological issues in the assessment of diabetes treatment adherence. Current Diabetes Reports, $11(6), 472-479$.

Haler, N. A., Gil, K. M., Gardner, W. G., \& Whittier, F. C. (2009). Patient computer use to prompt doctor adherence to diabetes management guidelines. Journal of Evaluation in Clinical Practice, 15(6), 1118-1124.

Haynes, R.B., Ackloo, E., Sahota, N., McDonald, H.P., \& Yao, X (2008). Intervention for enhancing medication adherence. The Cochrane Database of Systematic Reviews, 2, 23-38. Recuperado em 15 de setembro de 2011. Obtido em http://www.sefap.it/servizi_letteraturacardio_200807/CD000011.pdf

Holmbeck, G.N., Belvedere, M.C., Christensen, M., Czerwinski, A.M., Hommeyer J.S., Jonhson S.Z., \& Kung E. (1998). Assessment of adherence with multiple informants in pre-adolescents with spina bifida: Initial development of a multidimensional, multitask parent-report questionnaire. Journal of Personality Assessment, 70(3), 427-440.

Jonhson, S.B. (1992) Methodological issues in diabetes research: Measuring adherence. Diabetes Care, 15(11), 1658-1667.

Jonhson, S. B. (1993). Chronic diseases of childhood: Assessing compliance with complex medical componentens. In M. A. Krasnegor, L. Epstein, S. B. Jonhson, \& S. J. Yatte (Orgs), Developmental aspects of health compliance behavior (pp. 127-143). Hillsdale, NJ: Erlbaum.

Jonhson, S. B. (1995). Managing insulin-dependent diabetes mellitus in adolescence: A developmental perspective. In J. L. Wallander \& L. F. Siegel (Orgs.) Adolescent health problems: Behavioral perspectives. (pp.265-288). New York: Guilford Press. 
Karve, S., Pharm, M.S., Cleves, M.A., Helm, M., Hudson, T.J., West, D.S., \& Martin, B. C. (2008). An empirical basis for standardizing adherence measures for the assessment of diabetes self-management. Diabetes Care, 23, 1301-1304.

Keulen, H.M., Mesters, I., Mechelen, W., Vries, H. (2010). Single-item and multiple-item measures of adherence to public health behavior guidelines were incongruent. Journal of Clinical Epidemiology, 63, 75-84.

Kirkman, M., Williams, S., Caffrey, H., \& Marrero, D. (2002). Impact of a program to improve adherence to diabetes guidelines by primary care physicians. Diabetes Care, 25(11), 1946-1951.

Leite, S. N., \& Vasconcellos, M. P. (2003). Adesão à terapêutica medicamentosa: Elementos para a discussão de conceitos e pressupostos adotados na literatura. Ciência e Saúde Colectiva, 8(3), 775-782.

Lewin, A. B., Storch, E. A., Geffken, G. R., Heidgerken, A. D., Williams, L. B., \& Silverstein, J. H. (2005). Further examination of a structured adherence interview of diabetes for children, adolescents, and parents. Childrens Health Care, 34(2), 149-164.

Mackey, E.R., \& La Greca, A.M. (2007). Adolescents' eating, exercise, and weight control behaviors: Does peer crowd affiliation play a role? Journal of Pediatric Psychology, 31, 13-23.

Manzano, P., Pico, A., Serrano, J., Casimiro-Soriguer, F., Gonzalez, S., Tinahones, F., et al... (2006). Diabetes nutrition and complications trial: Adherence to the ADA nutritional recommendations, targets of metabolic control, and onset of diabetes complications. A 7-year, prospective, population-based, observational multicenter study. Journal of Diabetes and Its Complications, 20(6), 361-366.

Moraes, A. B.A., Rolim, G. S., \& Costa-Júnior, A. L. (2009). O processo de adesão numa perspectiva analítico comportamental. Revista Brasileira de Terapia Comportamental e Cognitiva, 11(2), 329-345. Recuperado em 15 de setembro de 2011 . Obtido em $<$ http://pepsic.bvsalud.org/scielo.php?script=sci_arttext\&pid=S1517$55452009000200009 \&$ Ing $=$ pt\&nrm $=$ iso $>$

Muller, A.D., Jaspan, H.B., Myer, L. Hunter, A.L., Harling, G., Bekker, L., \& Orrell. C. (2011). Standard measures are inadequate to monitor pediatric adherence in a resource-limited setting. AIDS Behavior, 15, 422-431 
Nemes, M.I.B., Helena, E.T.S., Caraciolo, J.M.M., \& Basso 1, C.R. (2009). Assessing patient adherence to chronic diseases treatment: Differentiating between epidemiological and clinical approaches. Cadernos de Saúde Pública, 25(3), 392-400.

Oliveira, B.M., Viana, M.B., Arruda, L.M., Ybarra, M.I. \& Romanha, A.J. (2005). Avaliação da adesão ao tratamento através de questionários: estudo prospectivo de 73 crianças portadoras de leucemia linfoblástica aguda. Jornal de Pediatria, 81(3), 245-250. Recuperado em 25 de setembro de 2013.

Obtido

em

http://www.scielo.br/scielo.php?script=sci_arttext\&pid=S002175572005000400012

Organização Mundial de Saúde [OMS] (2003) Adherence to long-term therapies: Evidence for action. Geneva: World Health Organization. Recuperado em 15 de setembro de 2013. Obtido em http://whqlibdoc.who.int/publications/2003/9241545992.pdf

Patton, S.R. (2006). Adherence to treatment in children and adolescents with type one diabetes mellitus. Special population pediatrics review, 1-4. Recuperado em 12 de agosto de 2011. Obtido em http://www.touchbriefings.com/pdf/2452/patton[1].pdf

Patton, S. R., Dolan, L. M., \& Powers, S. W. (2006). Mealtime interactions relate to dietary adherence and glycemic control in young children with type 1 diabetes. Diabetes Care, 29(5), 1002-1006.

Quittner, A. L., Espelage, D. L., Ievers-Landis, C. E., \& Drotar, D. (2000). Measuring adherence to medical treatments in childhood chronic illness: Considering multiple methods and sources of information. Journal of Clinical Psychology in Medical Settings, 7, 41-54.

Quittner, A.L., Modi, A., Lemanek, K.L., Ievers-Landis, C. E., \& Rapoff, M.A. (2008) Evidence-based assessment of adherence to medical treatments in pediatric psychology. Journal of Pediatric Psychology, 33(9), 916-936.

Rand, C.R. (1990). Issues in the measurement of adherence. In Sumaker, S.A., Schron, E.B., Ockene, J.K. (Orgs.). The handbook of health behavior change, (pp.102-110). New York: Springer Publishing Company.

Rapoff, M. A. (2009). Adherence to pediatric medical componentens. New York: Kluwer Academic/Plenum. 
Rudd, P. (1979). In search of the gold standard for compliance measurement. Archives of Internal Medicine, 139(6), 627-628.

Sackett, D.L. (1976). The magnitude of compliance and noncompliance. Em Sackett, D.L. \& Haynes, B.R. Compliance with therapeutic componentens. Johns Hopkings University Press: Baltimore.

Schilling, L.S., Grey, M., Knafl, K.A. (2002). A review of measures of selfmanagement of type 1 diabetes by youth and their parents. The Diabetes Educator, 28, 796-807.

Smith, H., Hankins, M., Hodson,A., \& George, C. (2010). Measuring the adherence to medication of elderly patients with heart failure: Is there a gold standard? International Journal of Cardiology, 145(1), 122-123.

Souza, A., Silva, D., Freitas, T., Borges, M. (2014) Avaliação do nível de atividade física em adolescentes com diabetes mellitus tipo 1 e sua correlação com variáveis metabólicas. Revista Brasileira de Atividade Física \& Saúde, 19(1), 109-120. Recuperado em 15 de julho de 2014, do Portal de Periodicos da UFPEL: http://www.periodicos.ufpel.edu.br/ojs2/index.php/RBAFS/article/view/298 5

Vitolins, M.Z., Rand, C.S., Rapp, S.R., Ribisl, P.M., \& Sevicky, A.M. (2000). Measuring adherence to behavioral and medical interventions. Controlled Clinical Trials, 21, 188-194.

Williams, G. C., Patrick, H., Niemiec, C. P., Williams, L. K., Divine, G., Lafata, J. E., et al... (2009). Reducing the health risks of diabetes: How selfdetermination theory may help improve medication adherence and quality of life. Diabetes Educator, 35(3), 484-492.

Yang, Y., Thumula, V., Pace, P. F., Banahan, B. F., Wilkin, N. E., \& Lobb, W. B. (2009). Predictors of medication nonadherence among patients with diabetes in medicare. Part D Programs: A retrospective cohort study. Clinical Therapeutics, 31(10), 2178-2188.

Contato: luzianek@yahoo.com.br, maluuel@gmail.com

Recebido em: 19/01/2014

Revisado em: 26/05/2014

Aceito em: $11 / 08 / 2014$ 